Majority $(80 \%)$ of planned imaging was for Brain relating to epilepsy or other neurodevelopmental disorders; other scans were booked for spine, ears or hips.

Results $67.2 \%$ children were successfully sedated and completed MRI scans (43/64).

Failure of sedation was $44 \%$ in older children (11/25) compared to $25.6 \%$ of those under 4 years age (10/39).

2 children vomited and 1 refused the oral medication.

18 children were not adequately sedated, of which 12 were re-booked for imaging with oral sedation and others booked for MRI under general anaesthetic.

2 patients also received oral Midazolam, of which 1 achieved successful MRI.

$18.6 \%$ of MRIs (8/43) were reported as poor quality or had movement artefacts, suggesting achievement of good quality imaging in 54.6\% planned elective MRIs (35/64).

There were no major complications but 2 children had low oxygen saturations related to deep sedation during scan; of which 1 child was kept overnight due to desaturations.

Conclusions Oral sedation using combination of Alimemazine Tartrate and Chloral Hydrate is safe within district general hospitals where provision of general anaesthetic is limited.

The partially successful sedation is also reported by other centres, leading to poor utilisation of MRI slots.

Inadequate sedation further impacts the quality of neuroimaging affecting diagnostic yield in potentially significant underlying neurodevelopmental disorders.

\section{COMPARING FAECAL TRANSMISSION PATHWAYS CONTRIBUTING TO ENTERIC INFECTIONS IN INFANTS IN RURAL INDIA}

Julia Vila-Guilera, Lena Ciric, Priti Parikh, Rajib Dasgupta, Monica Lakhanpaul. UK

\subsection{6/bmjpo-2021-RCPCH.69}

Background In rural India, child stunting remains a pressing concern that is being targeted by national flagship programmes. Poor child hygiene and exposure to enteric pathogens are important drivers of child stunting. Enteric pathogens can be transmitted from contaminated faeces to infants via water, food, hands, objects, soil, and flies.

Objectives In this study, we compared the infants' risk of enteric infection from exposure to different faecal transmission pathways, so that hygiene interventions can prioritise efforts towards the most dominant risks for infants.

Methods We collected data from 42 households with at least one infant aged 0 to 2 years from the study villages in rural Rajasthan, India. Water samples from drinking and bathing water sources, soil samples from household floors where infants were seen playing, and swabs from infants' and caregivers' hands were analysed for faecal indicator bacteria ( $E$. coli). Household observations and data from the literature on exposure assessments were used to determine the infant's frequency and level of exposure to these different faecal transmission pathways. Published ratios between E. coli and enteric pathogens were used to assess the risk of enteric infection from each different faecal transmission pathway analysed.

Results The transmission pathways analysed included: Mouthing of own infants' hands, mouthing caregivers' hands, direct ingestion of soil from the household floor, drinking household stored water, and involuntary ingestion of surface water during bathing events at local streams. Over $98 \%$ of all the samples tested were positive for faecal contamination. All of the surface water samples and household floor soil samples were highly contaminated with faecal bacteria $\left(>2 \log _{10}\right.$ CFU/100 $\mathrm{mL}$ and/g, respectively), and $93 \%$ of the drinking water samples were positive for E. coli (Geomean, SD 2.10 $\pm 0.76 \log _{10} \mathrm{CFU} / 100 \mathrm{~mL}$ ). Over $90 \%$ of the infants' and caregivers' hand swabs were contaminated. The direct ingestion of soil was the transmission pathway that posed that highest daily risk of enteric infection to infants, followed by mouthing of own soiled hands. Ingestion of soil posed a 1.4fold higher infection risk than drinking water. The involuntary ingestion of water while bathing at local streams and mouthing of caregivers' hands posed smaller infection risks, but still considerable after accumulating over time. After one year, the estimated risk of Campylobacter and enteropathogenic E. coli infection was $100 \%$ for all the analysed infection pathways.

Conclusions Water, sanitation and hygiene programmes have typically overlooked soil as a faecal exposure pathway, but results from this study highlight the need to prioritise reducing infants' exposure to faecally contaminated soil while crawling around the household floors and mouthing their own hands, as those pathways posed the highest infection risks. However, it is apparent that discrete hygiene interventions targeting individual pathways will not be enough to reduce the enteric infection burden, as all the transmission pathways analysed posed a high risk of infection over time. This study reinforces the need for transformative changes to address the overall widespread high levels of faecal contamination in the infants' living environment to reduce child stunting and achieve the Sustainable Development Goals.

\section{ROTA INNOVATION AND E-ROSTERING IN GENERAL PAEDIATRIC DEPARTMENT IN A LARGE DISTRICT GENERAL HOSPITAL}

Tom Cromarty, Pramodh Vallabhaneni. United Kingdom

\subsection{6/bmjpo-2021-RCPCH.70}

Background Junior Doctor rotas have gone through a number of iterations over the years. Rigid, rolling rotas with fixed annual and study leave have been opposed by trainees and the BMA1. They leave junior doctors unhappy, burnt out and disengaged. This has a negative effect on patient care ${ }^{3}$ and serious consequences for retention and recruitment in Paediatrics and Healthcare at large.

Objectives To introduce a new electronic rota management (HealthRota) system which will facilitate self-rostering, improve adherence to the BMA and $\mathrm{RCPCH}$ Trainee Charter 2 recommendations and improve junior doctor wellbeing.

Methods Twelve Junior Doctors (and senior doctor equivalents) starting a General Paediatric Medicine post will complete a survey of fourteen questions about their experiences in their previous rota (Non-HealthRota). They will then be introduced to HealthRota and repeat the survey towards the end of their Paediatric post. The survey included questions on life: work balance, rota design, transparency, access, leave requesting, annual leave, study leave, clinics and flexibility.

Results Overall there was on average a 1.3 point improvement (on a 5 point Leichardt scale) across all questions, with the various Non-HealthRota experiences having a satisfaction score of 3.0, whilst e-HealthRota had a score of 4.3. There were 